\title{
Avaliação dos hábitos alimentares em estudantes universitários
}

\author{
Evaluation of food habits in university students
}

\section{Evaluación de hábitos alimentarios en estudiantes universitarios}

\section{RESUMO}

Objetivo: Avaliar os hábitos alimentares de estudantes universitários. Método: Estudo descritivo, transversal, realizado com 204 universitários, por meio de questões adaptadas do instrumento para a Vigilância de Fatores de Risco e Proteção Para Doenças Crônicas por Inquérito Telefônico do Ministério da Saúde. Os dados foram analisados por meio de estatística descritiva. Resultados: Considerando o Guia Alimentar para a População Brasileira, encontrou-se um consumo considerado inadequado dos grupos alimentares: frutas, verduras e legumes, carboidratos, carnes e ovos, peixes, leite e derivados, frituras, doces, refrigerantes e sal, com consumo inadequado entre $60 \%$ a $96 \%$ dos universitários. Em relação a leguminosas, $61 \%$ consumiam, 63\% tiravam a gordura aparente das carnes, e 60\%, 99\% e 85\% realizavam as principais refeições café da manhã, almoço e jantar, respectivamente. Conclusão: Os hábitos alimentares entre os estudantes universitários apresentaram-se, em maior parte, inadequados, o que os predispõem a fatores de risco para o desenvolvimento de doenças cardiovasculares ao longo da vida. Esses dados colaboram para a importância de as universidades proporem ações de intervenção junto aos estudantes para escolhas mais saudáveis de alimentação.

Descritores: Dieta; Comportamento Alimentar; Estudantes.

\section{ABSTRACT}

Objective: To evaluate the eating habits of university students. Method: A descriptive, cross-sectional study conducted with 204 undergraduate students through questions adapted from the Risk Factors Surveillance and Protection for Chronic Diseases Survey instrument of the Ministry of Health. Data were analyzed using descriptive statistics. Results: Considering the Food Guide for the Brazilian Population, it was found an inadequate consumption of the food groups: fruits, vegetables, carbohydrates, meat and eggs, fish, milk and derivatives, fried foods, sweets, soft drinks, and salt, with inadequate consumption between $60 \%$ to $96 \%$ of university students. Results showed that $61 \%$ consumed vegetables (legumes), 63\% removed the apparent fat from meat, and $60 \%, 99 \%$, and $85 \%$ had the main meals, breakfast, lunch, and dinner, respectively. Conclusion: Eating habits among university students were mostly inadequate, which predispose them to risk factors for the development of cardiovascular diseases throughout their lives. These data contribute to the importance of universities proposing intervention actions with students for healthier food choices.

Descriptors: Diet; Eating Behavior; Students.

\section{RESUMEN}

Objetivo: Evaluar los hábitos alimenticios de los estudiantes universitarios. Método: Estudio descriptivo, transversal, realizado con 204 estudiantes universitarios a través de preguntas adaptadas del instrumento de Encuesta de Vigilancia de Factores de Riesgo y Protección para Enfermedades Crónicas del Ministerio de Salud. Los datos se analizaron mediante estadística descriptiva. Resultados: Considerando la Guía de Alimentos para la Población Brasileña, se encontró un consumo inadecuado de los grupos de alimentos: frutas, verduras, carbohidratos, carne y huevos, pescado, leche y derivados, alimentos fritos, dulces, refrescos y sal, con consumo inadecuado entre $60 \%$ a $96 \%$ de estudiantes universitarios. Con respecto a las legumbres, el $61 \%$ consumió, el $63 \%$ eliminó la grasa visible de la carne y el 60\%, 99\% y 85\% comió las comidas principales desayuno, almuerzo y cena, respectivamente. Conclusión: Los hábitos alimenticios entre los estudiantes universitarios fueron en su mayoría inadecuados, lo que los predispone a factores de riesgo para el desarrollo de enfermedades cardiovasculares a lo largo de sus vidas. Estos datos contribuyen a la importancia de que las universidades propongan acciones de intervención con los estudiantes para elegir alimentos más saludables.

Descriptores: Dieta; Conducta Alimentaria; Estudiantes.
Euripea Leite da Silva Oliveira ${ }^{1}$ (i) 0000-0002-0646-1698

Mara Cristina Ribeiro Furlan ${ }^{1}$ (D) 0000-0003-3227-7074

Catchia Hermes Uiliana ${ }^{1}$ (i) 0000-0002-2698-0022

Aires Garcia dos Santos Junior ${ }^{1}$ (D) 0000-0002-5946-0197

Letícia Akie Nagata ${ }^{1}$

(iD) 0000-0003-4418-086X

1 Universidade Federal de Mato Grosso do Sul.

Autor correspondente:

Euripea Leite da Silva Oliveira

E-mail: euripea.oliveira@hotmail.com

\section{Como citar este artigo:}

Oliveira ELS, Furlan MCR, Uiliana $\mathrm{CH}$, et al. Avaliação dos hábitos alimentares em estudantes universitários. Revista de Enfermagem do Centro Oeste-Mineiro. 2021;10:e3742. [Access___]; Available in: DOI: http://doi.org/10.19175/recom.v10i0.3742 


\section{INTRODUÇÃO}

A demanda por atendimento nutricional tem crescido, significativamente, tanto na Atenção Primária à Saúde quanto em clínicas e consultórios, em decorrência do aumento da prevalência de doenças crônicas não transmissíveis (DCNT) e do reconhecimento de que a adoção de uma dieta não saudável representa um dos principais determinantes dessas doenças ${ }^{(1)}$.

Aproximadamente, $82 \%$ das mortes prematuras por DCNT ocorrem em países de baixa e média renda, com estatísticas que demonstram que cerca de metade dessas mortes ocorrem, durante a vida produtiva dos indivíduos, sendo que as doenças cardiovasculares (DCV) são responsáveis pela maior parte delas, correspondendo a $37 \%{ }^{(2)}$.

Sabe-se que muitos dos fatores de risco cardiovasculares (FRC) são modificáveis, podendo contribuir para a diminuição da morbimortalidade quando controlados, dentre os quais estão incluídos a inatividade física e a alimentação não saudável ${ }^{(3)}$.

Uma alimentação, quando adequada e variada, contribui para a proteção contra as DCNT que são potencialmente fatais, como diabetes, hipertensão, acidente vascular cerebral (AVC), doenças cardíacas e alguns tipos de câncer que, em conjunto, estão entre as principais causas de incapacidade e morte no Brasil e em vários outros países ${ }^{(4)}$.

Por outro lado, a alimentação inadequada proveniente dos alimentos rápidos, em sua maioria, industrializados, prontos ou fáceis de preparar e não saudáveis, influenciam na baixa ingestão de frutas e hortaliças, redução do consumo de cereais integrais, leguminosas, raízes e tubérculos, tornando difícil conciliar saúde, aprendizado e prazer ${ }^{(5-6)}$.

Modificações provenientes da nova forma de vida, como a entrada na universidade, as novas relações sociais, a adoção de condutas e o estilo de vida diferenciado podem tornar os universitários grandes consumidores da alimentação inadequada e, possivelmente, um grupo vulnerável às circunstâncias que colocam em risco a sua saúde (7). Portanto, acredita-se que o acesso a informações sobre alimentação e nutrição e o monitoramento do consumo alimentar são importantes para a identificação de um comportamento de risco, bem como para garantir o pleno potencial de desenvolvimento nos universitários.
Na promoção da saúde, a relação existente entre a alimentação saudável e qualidade de vida vem sendo amplamente discutida nos últimos anos. Isso se deve ao fato de os estudos demonstrarem que a melhora dos hábitos alimentares pode reduzir o risco de desenvolvimento de diversas DCNT ${ }^{(8)}$.

Diante do papel fundamental que a alimentação saudável exerce na prevenção às DCV e, de que quanto mais precoce a adoção de hábitos saudáveis maior o fator de proteção na idade adulta ou idosa, este estudo objetivou avaliar os hábitos alimentares de estudantes universitários.

\section{MÉTODOS}

Foi realizado um estudo descritivo e transversal, desenvolvido junto a universitários de todos os cursos do Campus de Coxim (CPCX), da Universidade Federal de Mato Grosso do Sul (UFMS), quais sejam, Enfermagem, Letras, Sistemas de Informação e História.

O município de Coxim, do estado de Mato Grosso do Sul, na região Centro Oeste do Brasil, distante da capital Campo Grande 245 km, tem uma população prevista em 2017 de 33.323 habitantes ${ }^{(9)}$. É sede da microrregião Norte de saúde do Mato grosso do Sul, sendo referência para cinco municípios.

A coleta de dados foi realizada por uma equipe treinada, no período de julho a agosto de 2016, em sala de aula para cada turma, de maneira individual, após data pré-agendada junto ao coordenador de cada curso e autorização do professor que ministrava aula no momento. O critério de inclusão foi de estar matriculado, em curso de graduação da UFMS/CPCX. O critério de exclusão foi de estar cursando estágio curricular obrigatório. Todos os estudantes presentes em sala de aula foram convidados a participar. Havia 367 acadêmicos matriculados nos cursos da UFMS/CPCX. Desse total, 24 cursavam estágio curricular obrigatório. Houve uma recusa e 138 alunos não estavam presentes, na data agendada para coleta, sendo assim, 204 universitários participaram do estudo.

As orientações quanto aos objetivos da pesquisa, o caráter sigiloso das informações coletadas, o direito de recusar-se de participar em qualquer fase de execução, assim como a garantia de não sofrer qualquer ônus ou gratificação pela sua participação e sobre o preenchimento do instrumento, foram devidamente passadas aos universitários. O Termo de Consentimento Livre e 
Esclarecido (TCLE) foi assinado, em duas vias, pelos estudantes maiores de 18 anos, e os menores, obtiveram autorização do responsável por meio do Termo de Assentimento Livre Esclarecido (TALE), sendo que a coleta de dados para estes foi agendada para a data posterior.

O perfil socioeconômico foi avaliado por meio da Metodologia da Associação Brasileira de Empresas de Pesquisa ${ }^{(10)}$. A técnica agrupa as categorias, de acordo com o poder de compra das pessoas e famílias urbanas, a contratação de empregada doméstica e o grau de instrução do chefe da família. Os estudantes foram agrupados nas classes econômicas A1, A2, B1, B2, C1, C2, D e $E$, posteriormente reagrupadas em classes $A / B$ (alta), C (média) e D/E (baixa).

Ao estudar o consumo alimentar dos participantes, formulou-se um questionário com questões adaptadas do instrumento para a Vigilância de Fatores de Risco e Proteção Para Doenças Crônicas por Inquérito Telefônico (VIGITEL) do Ministério da Saúde ${ }^{(4)}$. As questões sobre consumo alimentar, permitiram uma resposta direta a cada alimento (frutas, verduras e legumes, feijão, alimentos gordurosos, açúcar, carne e refrigerante), sendo que as opções de resposta foram divididas em seis categorias (uma a duas vezes na semana, três a quatro vezes, cinco a seis vezes, todos os dias, quase nunca e nunca).

O consumo alimentar foi classificado em adequado e inadequado, segundo o guia alimentar brasileiro, que recomenda o consumo diário de seis porções de carboidratos, três porções de frutas, três porções de verduras e legumes, uma porção de leguminosas, uma porção de carne ou ovos, três porções de leite e derivados, uma porção dos alimentos do grupo de óleos e gordura, máximo $5 \mathrm{~g}$ de sal (uma colher rasa de chá), seis a oito copos de água. Além disso, deve-se ingerir peixe duas vezes, na semana e frituras, doces ou refrigerantes raramente ou nunca ${ }^{(11)}$.

Para análise, os dados foram transcritos para planilha eletrônica do Excel e verificados possíveis equívocos, como erros na entrada ou omissão de respostas e, posteriormente, foram empregadas estatísticas descritivas.

A pesquisa seguiu todos os aspectos éticos previstos na Resolução 466/12 do Conselho Nacional de Saúde. O projeto foi submetido via Plataforma Brasil (CAAE: 43290415.3.0000.0021) e aprovado pelo Comitê de Ética em Pesquisa com Seres Humanos da Universidade Federal de Mato Grosso do Sul, sob parecer número 1.065.671/2015.

\section{RESULTADOS E DISCUSSÃO}

Dos 204 participantes do estudo, observouse que $112(54,9 \%)$ eram estudantes do curso de Enfermagem, 48 (23,5\%) de Letras, 25 (12,3\%) de Sistemas de Informação e 19 (9,3\%) de História. Em relação às características sociodemográficas, 158 $(77,5 \%)$ eram do sexo feminino, 46 (22,5\%) do sexo masculino, $110(53,9 \%)$ se autodenominaram de cor parda, 61(29,9\%) branca, $16(7,8 \%)$ preta e quatro $(2,0 \%)$ amarela. Com relação à classe econômica, $135(66,2 \%)$ estudantes eram de classe econômica média, $39(19,1 \%)$ de classe alta e 29 $(14,3 \%)$ de classe baixa. A idade dos participantes variou de 17 a 58 anos, com média de 25,9 anos.

Tabela 1 - Frequência absoluta e relativa do consumo alimentar em estudantes universitários. Coxim, 2016

\begin{tabular}{llllll}
\hline Grupos de alimentos & \multicolumn{5}{c}{ Tipo de alimentação } \\
\cline { 2 - 6 } & $\mathbf{N}$ & Adequada & Inadequada & \% \\
\cline { 2 - 6 } Frutas & 44 & 21 & 160 & 79 \\
Legumes e verduras & 82 & 40 & 122 & 60 \\
Leguminosas & 124 & 61 & 80 & 39 \\
Carboidratos & 21 & 10 & 183 & 90 \\
Carnes e ovos & 58 & 28 & 146 & 72 \\
Peixes & 30 & 15 & 174 & 85 \\
Leite & 67 & 33 & 137 & 67 \\
Frituras & 38 & 19 & 166 & 81 \\
Doces e refrigerantes & 9 & 4 & 195 & 96 \\
Água & 121 & 60 & 83 & 40 \\
\hline
\end{tabular}

Fonte: Dados do estudo

Quanto ao consumo dos alimentos, na maior parte dos casos os estudantes universitários apresentaram consumo inadequado de frutas, legumes e verduras, em que 56 (27\%) não consomem todos os dias e 66 (32\%) consomem menos que o considerado adequado. $\mathrm{O}$ grupo das leguminosas foi o único que obteve como resultado, um consumo adequado em mais da 
4|Oliveira ELS, Furlan MCR, Uiliana CH, et al.

metade dos universitários, 14 (7\%) que não consomem e 66 (32\%) que consomem menos que o recomendado.

Em relação aos carboidratos, 38 (19\%) universitários consomem menos que 0 recomendado e 145 (71\%) consomem mais que o recomendado. Sobre carnes e ovos, 145 (71\%) consome além do que é considerado adequado. No que se refere ao leite e derivados, 8 (4\%) universitários não consomem, 31 (15\%) consome mais do que é adequado e 98 (48\%) menos que o recomendado. No que se refere às frituras, doces e refrigerantes, apenas uma pequena porcentagem consome, raramente, ou nunca tais alimentos (19\% e $4 \%$ respectivamente), sendo que a maioria respondeu entre "comem todos os dias ou mais que três vezes na semana" para ambos os grupos.

No tocante ao consumo regular de água, 33 (17\%) ingerem a quantidade recomendada, 88 (43\%) bebem até mais que oito copos por dia, enquanto que $83(40 \%)$ consomem menos que seis copos por dia (tabela 1).

Tabela 2 - Hábitos alimentares descritos pelos estudantes universitários. Coxim, 2016

\begin{tabular}{|c|c|c|}
\hline \multirow{2}{*}{ Consumo alimentar } & \multicolumn{2}{|c|}{ Total de estudantes } \\
\hline & $\mathrm{N}$ & $\%$ \\
\hline \multicolumn{3}{|l|}{ Costuma tirar a gordura aparente das carnes } \\
\hline Sim & 128 & 63 \\
\hline Não & 73 & 36 \\
\hline Não consome & 3 & 1 \\
\hline \multicolumn{3}{|l|}{ Tipo de leite e derivados } \\
\hline Integral & 136 & 67 \\
\hline Com baixo teor de gordura & 39 & 19 \\
\hline Não pasteurizado & 23 & 11 \\
\hline \multicolumn{3}{|l|}{ Tipo de gordura mais usado para cozinhar os alimentos } \\
\hline Gordura animal ou manteiga & 24 & 12 \\
\hline Óleo vegetal como: soja, girassol, milho, algodão ou canola & 169 & 83 \\
\hline Margarina ou gordura vegetal & 8 & 4 \\
\hline \multicolumn{3}{|l|}{ Coloca mais sal nos alimentos quando já servidos no prato } \\
\hline Sim & 172 & 84 \\
\hline Não & 32 & 16 \\
\hline \multicolumn{3}{|l|}{ Refeições que costuma fazer habitualmente no dia } \\
\hline \multicolumn{3}{|l|}{ Café da manhã } \\
\hline Sim & 122 & 60 \\
\hline Não & 82 & 40 \\
\hline \multicolumn{3}{|l|}{ Lanche da manhã } \\
\hline Sim & 54 & 26 \\
\hline Não & 150 & 74 \\
\hline \multicolumn{3}{|l|}{ Almoço } \\
\hline Sim & 202 & 99 \\
\hline Não & 2 & 1 \\
\hline \multicolumn{3}{|l|}{ Lanche ou café da tarde } \\
\hline $\operatorname{Sim}$ & 118 & 58 \\
\hline Não & 86 & 42 \\
\hline \multicolumn{3}{|l|}{ Jantar } \\
\hline $\operatorname{Sim}$ & 173 & 85 \\
\hline Não & 31 & 15 \\
\hline \multicolumn{3}{|l|}{ Lanche antes de dormir } \\
\hline $\operatorname{Sim}$ & 71 & 35 \\
\hline Não & 133 & 65 \\
\hline \multicolumn{3}{|c|}{$\begin{array}{l}\text { Costuma ler a informação nutricional que está presente no rótulo de alimentos industrializados } \\
\text { antes de comprá-los }\end{array}$} \\
\hline Nunca & 67 & 33 \\
\hline Quase nunca & 54 & 26 \\
\hline Algumas vezes & 65 & 32 \\
\hline Sempre ou quase sempre & 16 & 8 \\
\hline
\end{tabular}

Fonte: Dados do estudo. Resposta não sabe/não respondeu foram excluídas.

Houve prevalência de hábitos alimentares considerados adequados no que se refere à retirada de gordura aparente das carnes (63\%), assim como na utilização de óleos vegetais para o preparo dos alimentos (83\%). No entanto, observaram-se hábitos considerados inadequados 
como consumo de leite integral (67\%) em detrimento ao de baixo teor de gordura (19\%). Ademais, a maioria acrescentava sal nos alimentos já servidos no prato (84\%) (tabela2).

Quanto ao hábito de realizar uma das principais refeições, observou-se a frequência de 60\%, 99\% e 85\% para café da manhã, almoço e jantar, respectivamente (tabela 2).

A maioria dos estudantes (91\%) não tem um hábito rotineiro de ler rótulos de alimentos, antes de comprá-los.

\section{DISCUSSÃO}

Por meio dos dados apresentados, evidencia-se uma prevalência de hábitos alimentares considerados inadequados em mais da metade da amostra estudada de praticamente todos os grupos alimentares investigados. Apesar de estarem numa faixa etária jovem, a manutenção desses hábitos inadequados, os tornam indivíduos com fatores de risco para adquirirem doença cardiovascular.

Uma das hipóteses para esse comportamento alimentar entre estudantes é a de que, no cotidiano da vida universitária, a maioria está comprometida em ter um bom desempenho acadêmico e participar da vida cultural e social que a universidade proporciona, enquanto, para muitos, prover e cuidar da alimentação não é visto como algo de prioridade e importância ${ }^{(8)}$.

A ingestão dos grupos de alimentos frutas e verduras/legumes, que são fortes aliados à saúde como fatores de proteção para DCV, apresentaram valores de porção abaixo do recomendado 79\% e $60 \%$, respectivamente. Tais alimentos são considerados indispensáveis para uma alimentação saudável, pois são fontes de fibras, vitaminas, minerais, que estão associados à redução da pressão arterial, dos níveis sanguíneos de glicose e de lipídios ${ }^{(12)}$. Apesar da importância desses grupos alimentares, outros estudos também apontam consumo inadequado de frutas, verduras e legumes em estudantes universitários $^{(13-14)}$. O Estudo realizado em diferentes universidades do Chile, apontou que 94,3\% dos alunos não atenderam à recomendação ideal para o consumo de frutas, $72,3 \%$ para verduras e $97 \%$ para legumes ${ }^{(1)}$.

O consumo de leguminosas, neste estudo, foi positivo, atingindo um número considerado adequado em 61\% dos estudantes, o que é de fundamental importância, pois são as principais fontes de fibras benéficas para a função intestinal, ao reduzirem o tempo que o alimento leva para ser digerido e eliminado. Resultado positivo com relação ao consumo de leguminosas entre universitários foi descrito em diferentes estudos $^{(5,14)}$. Entretanto deve-se atentar que 39\% dos universitários, não consomem ou consomem menos que o recomendado, pois todas as leguminosas são fontes de proteína, vitaminas do complexo B e minerais, como ferro, zinco, cálcio e fibras, não devendo ser excluídas da alimentação.

O consumo inadequado de carboidratos atingiu $90 \%$ dos universitários, sendo que, estes consumiram quantidades superiores ao recomendado de bolos, pães, bolachas e menor consumo de arroz, milho e outros cereais, raízes e tubérculos. Os resultados são condizentes com a Pesquisa de Orçamento Familiar do Brasil, que mostrou uma elevação no consumo de alguns alimentos ricos em carboidratos simples, como biscoitos, bolos e refrigerantes, porém houve uma queda no consumo de carboidratos complexos na população em geral|(14).

Os carboidratos são subdivididos em carboidratos complexos (amidos) como: cereais, tubérculos e raízes; e carboidratos simples (açúcares simples ou livres) como: açúcar de mesa, refrigerantes, sucos artificiais, doces e guloseimas em geral. A alimentação saudável deve incluir os carboidratos complexos em maior quantidade, pois são fonte de energia e vitaminas do complexo $\mathrm{B}$ e ácidos graxos essenciais que participam do metabolismo do sistema nervoso. Os carboidratos simples devem compor a alimentação em quantidades bem reduzidas, já que são fontes apenas de energia e o seu consumo excessivo está relacionado com o aumento de risco de obesidade, cáries dentais e outras DCNT ${ }^{(11)}$

Em relação à ingestão de carnes, ovos e peixe, os resultados não foram satisfatórios, pois se evidenciou um consumo além do recomendado para carnes e ovos e um consumo abaixo do preconizado para peixes. O estudo corrobora com esses achados ${ }^{(14)}$, e demonstrara alto consumo de carne e ovos nessa população.

As carnes vermelhas são excelentes fontes de proteína de alta qualidade e têm teor elevado de muitos micronutrientes, especialmente ferro, zinco e vitamina B12. Porém, tendem a ser ricas em gorduras em geral, especialmente, as gorduras saturadas, que, quando consumidas em excesso, aumentam o risco de DCV e de várias outras doenças crônicas. Os ovos são ricos em proteínas de alta qualidade, minerais e vitaminas, como as do complexo B, componentes de uma alimentação 
saudável, desde que consumidos com moderação, de acordo com a orientação dada para todos os alimentos de origem animal. Por sua vez, os peixes são ricos em proteína de alta qualidade, contém vitaminas e minerais, além de conter menor conteúdo de gorduras saturadas e alta proporção de gorduras saudáveis (gorduras insaturadas), por tantas qualidades, seu consumo é recomendado pelo menos duas vezes ou mais por semana ${ }^{(11)}$.

No que se refere ao consumo de leite, chama a atenção que $48 \%$ dos estudantes relataram consumir um ou menos que um copo de leite por dia, dado preocupante, pois, sabe-se que, esse grupo alimentar é importante fonte de vitaminas e minerais, principalmente o cálcio, e oferece proteínas de excelente valor biológico, ainda mais quando a população estudada já apresenta baixo consumo de frutas, verduras e legumes. E quanto ao tipo de leite utilizado entre os que consomem, prevaleceu o do tipo integral. Os tipos e as quantidades desses alimentos devem ser adequados às diferentes fases do curso da vida. Leites e derivados devem ser preferencialmente desnatados para os adultos, pois contém menor teor de gordura e mesma quantidade de cálcio, e os integrais para crianças, adolescentes e gestantes $^{(15-16)}$.

O consumo elevado de frituras, doces e refrigerantes observados no presente estudo, foi evidenciado em outros trabalhos anteriores ${ }^{(8,13-17)}$. O consumo desses alimentos nas últimas décadas aumentou muito em detrimento do consumo de alimentos básicos in natura que têm sido substituídos por alimentos processados em decorrência do modo de vida contemporâneo, além de apresentarem alta densidade energética, serem ricos em açúcar, sódio e gorduras, favorecem o aumento da obesidade e doenças associadas $^{(18)}$.

Em relação ao consumo de água, o resultado foi satisfatório, pois ingerir água diariamente e na proporção certa é essencial para manutenção da vida, sendo que a quantidade recomendada é extremamente simples: a quantidade que nosso organismo (ou nossa sede) pedir(11).

Com relação aos hábitos alimentares descritos pelos universitários, mais de 50\% dos estudantes retiram a gordura aparente das carnes, que é um hábito considerado saudável, pois a recomendação é dar preferência às carnes magras, que contém baixo teor de gordura, assim como retirar a gordura aparente das carnes antes do consumo, minimizando o risco de desenvolvimento de doenças cardíacas ${ }^{(16)}$.
O hábito inadequado de acrescentar mais sal nos alimentos quando já servidos no prato, foi relatado por $83 \%$ dos estudantes deste estudo. Resultado condizente com Análise do Consumo alimentar Pessoal no Brasil(14), em que se observou uma proporção de indivíduos com ingestão de sódio acima do nível seguro, de $89 \%$ entre os homens e de $70 \%$ entre as mulheres para a faixa etária de 19 a 59 anos. Os dados são preocupantes, pois, o sódio é considerado um importante marcador da qualidade da alimentação, o consumo diário de sal deve ser no máximo de $5 \mathrm{~g} / \mathrm{dia}^{(16)}$. Essa quantidade é suficiente para atender às necessidades de iodo-essencial para o desenvolvimento e crescimento humano. O sal de cozinha (cloreto de sódio) é composto por $40 \%$ de sódio que, quando consumido em excesso, é uma causa importante da hipertensão(19). Em um trabalho realizado com estudantes de uma universidade, encontraram resultado com semelhança e divergência em que $66,45 \%$ dos estudantes retiram a gordura aparente das carnes, e $75,48 \%$ não adicionam sal nas refeições ${ }^{(20)}$.

A preocupação em torno da elevada ingestão do sal tem se tornado um assunto discutido em nível mundial. Segundo a OMS, procurar medidas a fim de reduzir a ingestão de sal é uma das principais maneiras dos países melhorarem a situação de saúde da população, ainda mais quando se considera que a redução de sua ingestão é a medida mais custo-efetivo para diminuir a mortalidade por DCV. Caso o consumo de sal fosse reduzido para menos de $5 \mathrm{~g}$ por dia, conforme recomendado, cerca de 2,5 milhões de mortes poderiam ser evitadas ${ }^{(21)}$.

Em relação às refeições realizadas, mais de $50 \%$ dos estudantes realizam as três refeições principais (dejejum, almoço e jantar), e um número razoável de estudantes consome os lanches intermediários. Resultados condizentes com o recomendado, que orienta que cerca de $90 \%$ do total de calorias consumidas, ao longo do dia, sejam provenientes das três refeições principais, café da manhã, almoço e jantar, destacam-se a importância de não negligenciar essas refeições e intercalar com pequenos lanches ${ }^{(11)}$. No entanto, o estudo demonstrou que é comum estudantes universitários negligenciarem alguma das principais refeições e realizarem lanches entre elas, geralmente consumindo alimentos com baixa densidade de nutrientes, como lanches ou sanduíches, vendidos em lanchonetes ${ }^{(8)}$.

A falta de tempo para fazer pequenos lanches, a indisponibilidade de alimentos 
saudáveis e a ausência do hábito de levar alimentos de casa para o trabalho, são algumas das causas mais frequentes de erros alimentares relacionados ao fracionamento das refeições. Muitos estudos mostram que uma alimentação fracionada, ao longo do dia, auxilia na redução da fome e evita a compensação nas refeições seguintes, apontando para uma relação inversa entre o peso corporal e adiposidade e a frequência das refeições ${ }^{(22)}$.

O óleo vegetal foi o tipo de gordura utilizado para cozinhar pela maioria dos estudantes, resultado considerado positivo, pois 0 recomendado é dar preferência aos óleos vegetais e azeite. No presente estudo, não foi investigado a quantidade diária utilizada, sendo a ideal de uma porção do grupo de óleos e gorduras ${ }^{(16)}$. Em outro estudo se obteve utilização de óleo vegetal pela maioria dos estudantes $(75,48 \%)^{(20)}$.

$\mathrm{Na}$ nutrição humana, os óleos têm importância significativa, uma vez que são ricos em ácidos graxos insaturados (ácido oléico, ácido linoléico e alfa-linoléico) e, pobres em ácidos graxos saturados, além de serem veiculadores de vitaminas lipossolúveis e fornecedores de energia. $O$ cuidado se deve à quantidade utilizada e a fritura que leva a alterações físico-químicas nos óleos, que pode provocar a perda do valor nutritivo e formar compostos prejudiciais para o organismo ${ }^{(23)}$. 0 consumo elevado de ácidos graxos e gorduras trans presente nas frituras, pode levar a dislipidemias, obesidade e aumentar o risco de DCV(11).

Quanto aos rótulos dos alimentos industrializados, somente $8 \%$ dos estudantes relataram ler sempre ou quase sempre as informações nutricionais antes de comprá-los. As informações contidas nos rótulos podem ser muito úteis na escolha de alimentos mais saudáveis, e, uma maneira de incentivar a consulta é promover a compreensão das informações, e a utilização dos rótulos como material em atividades didáticas em salas de aula, centros de saúde, centros comunitários e centros de convivência. Os profissionais de saúde e da educação devem procurar oportunidades para promover grupos para essa discussão ${ }^{(16)}$.

No presente estudo, foram revelados hábitos inadequados, em vários grupos alimentares entre os estudantes universitários, assim como em outros estudos similares. Esses resultados podem estar associados à mudança de ambiente o que implica responsabilidade, socialização, novas tarefas, excesso de atividades, priorização de desempenho acadêmico, entre outros fatores que se traduz num processo de adaptação vivenciada pelo indivíduo no egresso a uma universidade. Esses hábitos inadequados poderão permanecer após a formatura, sendo um sério fator de risco cardiovascular nesses estudantes, causando consequências danosas a saúde.

A impossibilidade de retorno à sala de aula, para coleta de dados de acadêmicos que não estavam presentes no primeiro momento ou equipe disponível para captar os que estavam no estágio curricular obrigatório, são fatores limitantes do estudo. Assim como a ausência da coleta de características pessoais que poderiam estar relacionadas com os hábitos alimentares.

\section{CONCLUSÃO}

Conclui-se que os estudantes universitários analisados, no presente estudo, apresentaram hábitos alimentares considerados como inadequados, na maioria dos parâmetros avaliados. Os dados apresentados permitiram identificar algumas situações que são fatores de risco cardiovasculares como: baixo consumo de peixes, frutas, verduras e legumes, consumo excessivo de carboidratos simples, carne, leite integral em detrimento ao de baixo teor de gordura e o consumo do sal em excesso.

Evidencia-se a importância e a necessidade de uma atuação preventiva e de promoção de saúde junto aos estudantes, por parte das universidades, com ações que priorizem uma conscientização de que sem hábitos preventivos, dentre as quais, uma alimentação adequada, não se obtém uma redução de risco e das consequências futuras a saúde, além de proporcionar melhor qualidade de vida, inclusive melhor rendimento nos estudos. Torna-se necessário o desenvolvimento de estratégias que resultem em formandos conscientes da importância de adotar medidas preventivas, ao longo da vida, para a manutenção da saúde, e assim também ganhar mais disseminadores de hábitos saudáveis para a população.

\section{REFERÊNCIAS}

1 - Vera $V$, Crovetto $M$, Valladares $M$, Oñate $G$, Fernández M, Espinoza $V$, et al. Consumo de frutas, verduras y legumbres en universitarios chilenos. Rev Chil Nutr. 2019;46(4):436-42. DOI: $\underline{10.4067 / \text { s0717-75182019000400436 }}$ 
2 - Siqueira ASE, Siqueira-Filho AG, Land MGP. Análise do impacto econômico das doenças cardiovasculares nos últimos cinco anos no Brasil. Arq Bras Cardiol. 2017;109(1):39-46. DOI: 10.5935/abc.20170068

3 - Santos JS, Patricio ACFA, Alves KL, Albuquerque KF, Pereira IL, Félix IVB. Avaliação para riscos cardiovasculares em estudantes de enfermagem. Rev Min Enferm. 2015;19(4):842-7. DOI: $\underline{10.5935 / 1415-2762.20150065}$

4 - Ministério da Saúde (Brasil). Vigitel Brasil 2017 Saúde Suplementar: Vigilância de fatores de risco e proteção para doenças crônicas por inquérito telefônico. Brasília: Ministério da Saúde; 2017.

5 - Mier GM, Estevan MCL, Magdalena CSR, Diego JP, Herreros PV. Evaluación del consumo de alimentos de una población de estudiantes universitarios y su relación con el perfil académico. Nutr Hosp. 2017;34(1):134-43. DOI: $\underline{10.20960 / \text { nh. } 989}$

6 - Monteiro CA, Cannon G, Levy RB, Moubarac JC, Jaime $P$, Martins AP, et al. NOVA. A estrela brilha. World Nutrition 2016 [citado em 14 abr 2020]; 7(13):28-40. Acesso em: http://archive.wphna.org/wp-

content/uploads/2016/02/WN-2016-7-1-3-28-40Monteiro-Cannon-Levy-et-al-NOVA-

$\underline{\text { Portuguese.pdf }}$

7 -Rosa PBZ, Giusti L, Ramos M. Educação alimentar e nutricional com universitários residentes de moradia estudantil. Ciênc Saúde 2016;9(1):15-20. DOI: $\quad$ 10.15448/1983$\underline{652 X .2016 .1 .20852}$

8 - Bernardo GL, Jomori MM, Fernandes AC, Proença RPC. Food intake of university students. Rev Nutr. 2017; 30(6):847-65. DOI: 10.1590/1678$\underline{98652017000600016}$

9 - Instituto Brasileiro de Geografia e Estatística. Estimativas da população residente para os municípios e para as Unidades da Federação brasileiros com data de referência em 1 o de julho de 2017. Rio de Janeiro: IBGE; 2017 [citado em 16 out 2019]. Acesso em: https://biblioteca.ibge.gov.br/visualizacao/livros/li v100923.pdf

10 - Associação Brasileira de Empresas de Pesquisa. Critério de Classificação Econômica Brasil. São Paulo: ABEP; 2014 [citado em 8 dez
2020]. Acesso em: http://www.abep.org/criterio$\underline{\text { brasil }}$

11 - Ministério da Saúde (Brasil). Secretaria de Atenção à Saúde. Guia alimentar para a população brasileira: Promovendo a alimentação saudável. 2. ed. Brasília: Ministério da Saúde; 2014.

12 - Silva HS, Silva LIA, Silva NBP, Prado BG. Consumo de fibras alimentares por universitários de Várzea Grande - Mato Grosso. J Health Biol Sci. 2019 [citado em 14 abr 2020]; 7(3):248-52. Acesso em:

http://docs.bvsalud.org/biblioref/2019/07/10056 33/2446-9852-4-pb.pdf

13 - Perez PMP, Castro IRR, Franco AS, Bandoni DH, Wolkoff DB. Práticas alimentares de estudantes cotistas e não cotistas de uma universidade pública brasileira. Ciênc Saúde Coletiv 2016;21(2):531-42. DOI: $\quad$ 10.1590/1413$\underline{81232015212.01732015}$

14 - Carneiro MNL, Lima PS, Marinho LM, Souza MAM. Estado nutricional de estudantes associados aos hábitos alimentares. Rev Soc Bras Clín Méd. 2016 [citado em 16 out 2019]; 14(2):84-8. Acesso em:

http://docs.bvsalud.org/biblioref/2016/08/1248/1 $\underline{4284 . p d f}$

15 - Instituto Brasileiro de Geografia e Estatística. Pesquisa de orçamentos familiares 2008-2009: Análise do consumo alimentar pessoal no Brasil. Rio de Janeiro: IBGE; 2011.

16 - Ministério da Saúde (Brasil). Guia alimentar para a população brasileira: Promovendo a alimentação saudável. Brasília: Ministério da Saúde; 2008.

17 - Busato MA, Pedrolo C, Gallina LS, Rosa L. Ambiente e alimentação saudável: Percepções e práticas de estudantes universitários. Sem, Cienc Biol Saúde 2015;36(2):75-84. DOI: 10.5433/1679$\underline{0367.2015 \mathrm{v} 36 \mathrm{n} 2 \mathrm{p} 75}$

18 - Pastor R, Bibiloni MM, Tur MJA. Patrones de consumo de alimentos en studiantes universitarios de Zamora. Nutr Hosp. 2017;34(6):1424-31. DOI: $\underline{10.20960 / \text { nh.1147 }}$

19 - Bucharles SGE, Wallbach KKS, Moraes TP, Pecoits-Filho R. Hipertensão em pacientes em diálise: Diagnóstico, mecanismos e tratamento. J 
Bras Nefrol. 2019; 41(3):400-11. DOI: $\underline{10.1590 / 2175-8239-j b n-2018-0155}$

20 - Mendes MLM, Silva FR, Messias CMBO, Carvalho PGS, Silva TFA. Hábitos alimentares e atividade física de universitários da área de saúde do município de Petrolina-PE. Tempus 2016 [citado em 16 out 2019]; 10(2):205-17. Acesso em: http://www.tempusactas.unb.br/index.php/temp us/article/view/1669

21 - Organização Mundial da Saúde (OMS). Folha informativa - Alimentação saudável [citado em 29 out 2020]. Acesso em: https://www.paho.org/bra/index.php?option=co $\underline{m}$ joomlabook\&view=topic\&id $=227$

22 - Generoso Junior AC, Silveira JQ. A influência do acompanhamento nutricional para a redução de gordura corporal e aumento de massa magra em mulheres praticantes de treinamento funcional. RBNE 2017 [citado em 14 abr 2020]; 11(64):485-93. Acesso em: http://www.rbne.com.br/index.php/rbne/article/v $\underline{\text { iew/839 }}$

23 - Jorge N, Pietro TA, Luzia DMM, Veronezi CM. Caracterização fitoquímica do óleo de soja adicionado de extrato de Portulaca oleracea L. Rev Ceres 2018;65(1):1-6. DOU: 10.1590/0034737×201865010001
Nota: Este estudo é resultado de um projeto de pesquisa maior intitulado "Prevalência de fatores de risco cardiovascular em universitários" e do Trabalho de Conclusão de curso intitulado "Avaliação dos hábitos alimentares em estudantes universitários de Coxim-MS" do curso de graduação em Enfermagem da Universidade Federal de Mato Grosso do Sul, campus Três Lagoas.

Recebido em: 20/04/2020

Aprovado em: $14 / 10 / 2020$ 